\title{
Wichtige Themen der Beratung, Versorgung und Forschung
}

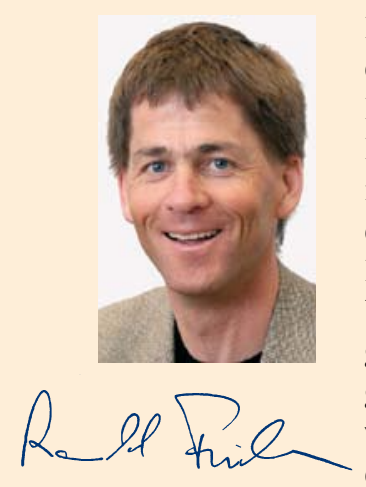

Liebe Kolleginnen und Kollegen,

durch die Rotation der Autoren für das Editorial ist es häufig an der BExMed, das Herbsteditorial zu verfassen. Wie immer im Herbst, und das ist besonders von München aus zu beobachten, ziehen am Wochenende die Ströme der Bergsteiger ins Gebirge. Herbstzeit scheint also Bergzeit zu sein. Das gilt übrigens nicht nur für die Alpen, sondern auch für viele Gegenden im Himalaja und in Südamerika.

Im Journal-Club der BExMed greift Helga Vollendorf diesmal ein Thema auf, das beim Bergsteigen oft ignoriert wird oder aber zu häufigen, oft falschen Ratschlägen führt: Höhenaufenthalt von Kindern. Da es hier nicht sehr viele Publikationen gibt, hebt sie eine Arbeit von 1998 hervor, die besonders junge Patienten im Alter von 3 bis 36 Monaten untersucht hat. Im Kommentar zu diesem Journal-Club finden Sie auch den Childrens Lake Louise Score, der die Beurteilung der Schwere der Höhenkrankheit auch für Kinder ermöglicht. Denn auch kleine Kinder können durchaus in die Höhe, wenn entsprechende Vorsichtsmaßnahmen und Spielregeln eingehalten werden. Die Literatur zu dem Consensus Statement der International Society of Mountain Medicine finden Sie ebenfalls in diesem Beitrag. Von der Höhen hinunter auf Meereshöhe bringt uns die Kasuistik des Schiffsarztes Tim Lammerding zur Behandlung eines Stichs mit einem giftigen Meerestier nicht an Bord des Kreuzfahrtschiffes, sondern am Strand während eines Strandtages. Diese Kasuistik zeigt erneut auf, wie vielfältig die Anforderungen an einen Schiffsarzt sind.

Dazu passt der Beitrag von Christoph Sevenich, der den Stellenwert von automatisierten, elektrischen Defibrillatoren (AED) an Bord nicht nur von Kreuzfahrtschiffen, sondern auch bei Handelsschiffen beleuchtet. Denn trotz der relativ geringen Zahl von betroffenen Personen kann ein AED aufgrund der speziellen Umstände eine sehr sinnvolle Ausstattung an Bord sein.

Erfreulicherweise gibt es in der Impfstoffentwicklung derzeit einige neue Zulassungen, die jeder Leser der FTR kennen sollte. Die jetzt verfügbaren neuen Meningokokkenimpfstoffe stellen die Kollegen Christof Vinnemeier und Jakob Cramer von der Tropenmedizin Hamburg vor.

Im Panoramateil schließlich weisen Oliver Ullrich und Jürgen Graf auf einen Teil der medizinischen Forschung hin, der leider viel zu oft vergessen wird: der Transport der Probe vom Ursprungsort ins Labor. Dies ist ja schon im täglichen Alltag der medizinischen Versorgung ein häufiges und leidiges Problem, wenn durch ,unglückliche‘ Umstände Blut-, Sputum- oder Gewebeproben nicht zeitgerecht und lagerungsgerecht im Labor eintreffen. Um wie viel schwerer wiegt es, wenn Proben, die in aufwendigen Messkampagnen gewonnen werden, durch banale Umstände wie technische Defekte am Transportmittel untauglich werden und damit Monate oder Jahre der Vorbereitung zunichte gemacht werden. Mit diesem kurzen Überblick auf das vorliegende Heft grüße ich Sie herzlich aus München und verbleibe mit den besten Wünschen für einen ruhigen Jahresausklang.

Ihr Rainald Fischer, München 